\title{
Two-Dimensional Echocardiographic Imaging of Distal Right Coronary Artery in Kawasaki Disease
}

\author{
Zia Q. Farooki, MD, Sandra K. Clapp, MD, William L. Jackson, MD, \\ Burton L. Perry, MD, and Edward W. Green, MD
}

Kawasaki disease, or mucocutaneous lymph node syndrome, is a common febrile illness affecting young children. It is characterized by fever, lymphadenitis, mucosal and cutaneous inflammation, and vasculitis. It is usually a self-limiting disease. Approximately $15 \%$ of Japanese children with Kawasaki disease develop coronary arteritis. ${ }^{1}$ Development of coronary artery aneurysms and thrombosis may result in death in $1 \%$ of the cases. ${ }^{1}$ Spontaneous resolution of coronary artery aneurysms has been documented in $50 \%$ of the cases. ${ }^{1}$ Attempts have been made to identify children who are at risk of developing coronary artery aneurysms. A scoring table introduced by Asai has not proved useful in many reported cases. $^{2,3}$ Initially, coronary angiography was felt to be the only definitive way of evaluating the coronary arteries but recently, two-dimensional echocardiography has proved useful in identifying aneurysms of the main right and left coronary arteries. ${ }^{4-7}$ Some studies have stressed that aneurysms of the distal right and left coronary arteries cannot be visualized by echocardiography., Yoshida et al. may be the first authors to report visualization of peripheral right coronary artery aneurysms by subcostal two-dimensional echocardiography. ${ }^{8}$

We would like to present our findings in two cases of Kawasaki disease in whom peripheral right coronary artery aneurysms were first diagnosed by two-dimensional echocardiography.

From Section of Cardiology, Children's Hospital of Michigan \& Wayne State University School of Medicine, Detroit, Mich. Manuscript received May 9, 1983; revised manuscript accepted November 14, 1983. For reprints contact Zia Q. Farooki, MD, 3901 Beaubien Blvd., Children's Hospital of Michigan, Detroit, Mich. 48201.

\section{CASE REPORTS}

Case 1

A 7-year-old white boy was admitted with the diagnosis of Kawasaki disease. He had no clinical signs of cardiac disease. His maximum erythrocyte sedimentation rate was $58 \mathrm{~mm} /$ hour. His platelet count was $405,000 / \mathrm{mm}^{3}$. He was treated with acetylsalicylic acid. Serial chest roentgenograms during the hospital stay showed mild cardiac enlargement of nonprogressive nature (cardiothoracic ratio $=0.5$ ). Serial electrocardiograms did not show any chamber enlargement or ST-T wave changes. Initial M-mode echocardiogram showed minimal left atrial enlargement, minimal left ventricular enlargement, normal left ventricular systolic shortening fraction, normal aortic pre-ejection period/ejection time ratio, normal ventricular septal motion, and thickening and a small posterior pericardial effusion. A twodimensional echocardiogram did not show any coronary artery aneurysms. The Asai score was 6. The patient was discharged after 10 days of hospitalization. A two-dimensional echocardiographic examination done 2 months after the onset of the illness showed bilateral coronary artery aneurysms. This examination was performed with Advanced Technology Laboratories mechanical sector scanner, utilizing a $5 \mathrm{MHz}$ transducer, in the parasternal, apical, subcostal, and suprasternal positions. The images were displayed according to the recommendations of the American Society of Echocardiography. The aneurysms of the right coronary artery were best visualized in the subcostal views (Figs. 1 and 2). These findings were confirmed by aortography (Fig. 3). The patient was treated with aspirin and remains asymptomatic 18 months after the onset of the disease. 


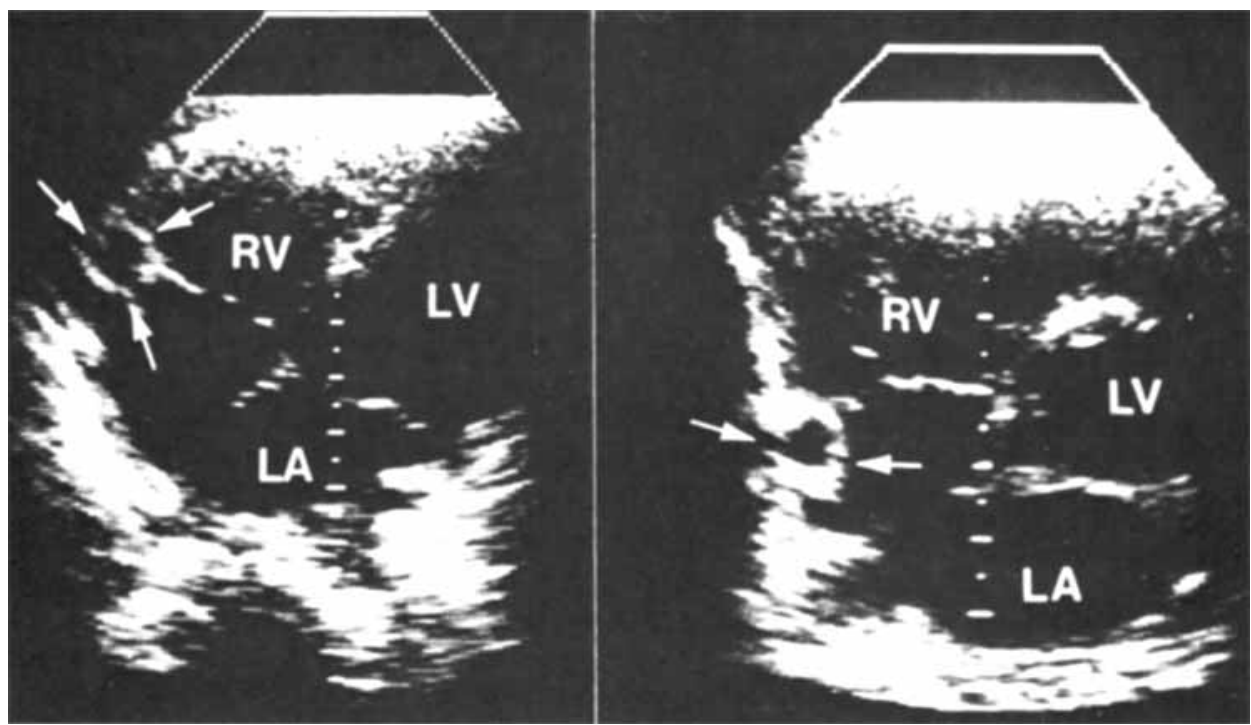

FIGURE 1. Echocardiogram: a subcostal 4-chamber view showed a circular echo-free space ( 3 white arrows) in the region of the tricuspid annulus. With additional angulation and rotation, it was seen as a circular echofree space along the right atrial wall (2 white arrows). This was felt to represent an aneurysm of the right coronary artery in the proximal right atrioventricular groove. $L A=$ left atrium, $L V=$ left ventricle, $R V=$ right ventricle.

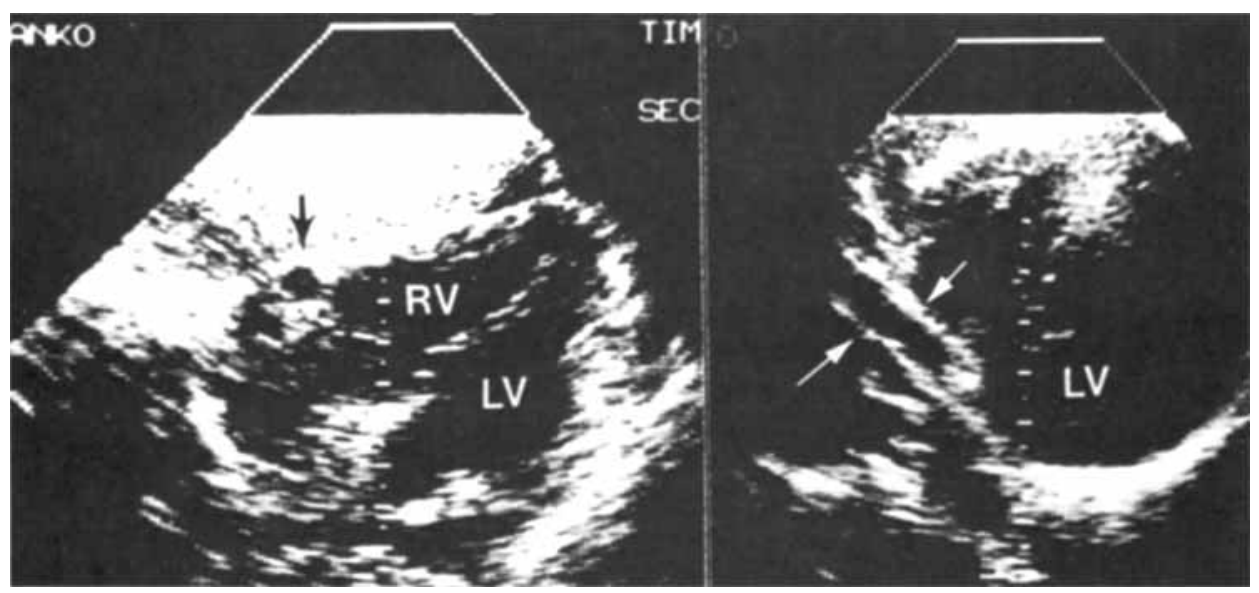

FIGURE 2. Echocardiogram: a subcostal 4-chamber view showed aneurysmal dilatation of right coronary artery (black arrow). With additional angulation and rotation of the transducer posteriorly, a dilated distal right coronary artery was seen as a tubular echo-free structure (2 white arrows). Abbreviations as in Fig. 1.

\section{Case 2}

An 18-month-old white girl was admitted with the diagnosis of Kawasaki disease. There were no clinical signs of cardiac disease during the hospital stay. The maximum erythrocyte sedimentation rate was $63 \mathrm{~mm} /$ hour. The highest platelet count was $793,000 / \mathrm{mm}^{3}$. Serial electrocardiograms showed transient nonspecific $\mathrm{T}$-wave flattening. Multiple chest roentgenograms showed normal heart size. Initial M-mode echocardiogram showed normal chamber dimensions, normal left ventricular shortening fraction, normal pre-ejection period/ejection time ratio, normal ventricular septal motion, and no pericardial effusion. A two-dimensional echocardiogram showed no coronary artery aneurysms. The Asai score was 3 . After 10 days of hospitalization, the patient was discharged on aspirin treatment. A follow up two-dimensional echocardiogram done 3 months after the onset of the disease showed coronary artery aneurysms (Fig. 4). These findings were confirmed by aortic angiography (Fig. 5). The patient remains asymptomatic on aspirin treatment 6 months after the onset of the disease. 


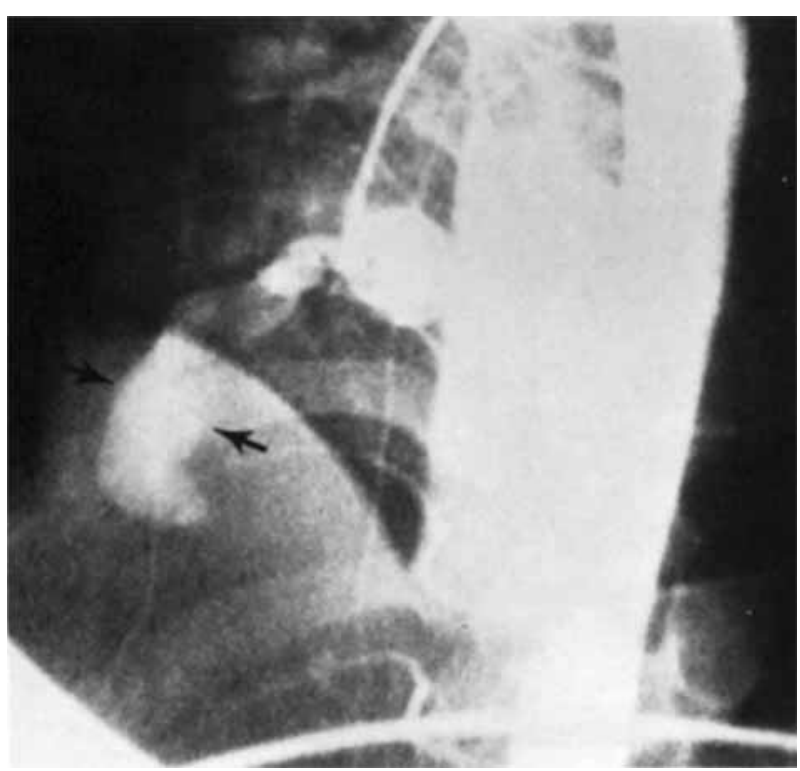

FIGURE 3. Aortogram: massive dilatation of the entire right coronary artery (arrows) was seen. There was marked stasis of the dye and poor filling of the small branches of the artery.

\section{DISCUSSION}

Kawasaki disease, or mucocutaneous lymph node syndrome, has been diagnosed with increasing frequency over the last 5 years. Its major importance is the possibility of damage to the coronary arteries. For this reason, it is of great importance to develop noninvasive method of identifying those children who will or have developed coronary artery involvement during the course of this illness. Asai et al. developed a scoring system which was designed to identify patients at higher risk for developing coronary artery complications. $^{2}$ They recommended coronary arteriography if the patient had a high score. Our two patients and others ${ }^{3}$ scored low but still developed

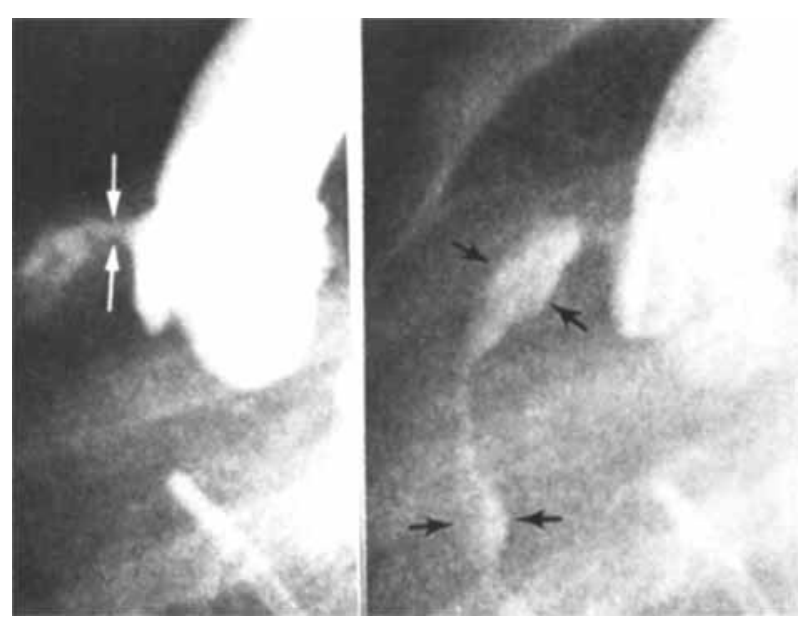

FIGURE 5. Aortogram: the proximal right coronary artery was normal at its origin (white arrows) but multiple aneurysms involved the distal right coronary artery (black arrows).

coronary artery complications. Recently, twodimensional echocardiographic reports have stressed that peripheral right coronary artery aneurysms may not be visualized by echocardiography. However, our experience confirms the findings of Yoshida et al. that two-dimensional subcostal echocardiography can detect aneurysms of the distal right coronary artery. A circular echo-free space in the area of the right atrioventricular groove is highly suggestive of aneurysmal dilatation of the right coronary artery. We have not encountered this finding in any normal infant or child. This approach should prove useful in the early diagnosis of the right coronary artery aneurysms in Kawasaki disease.

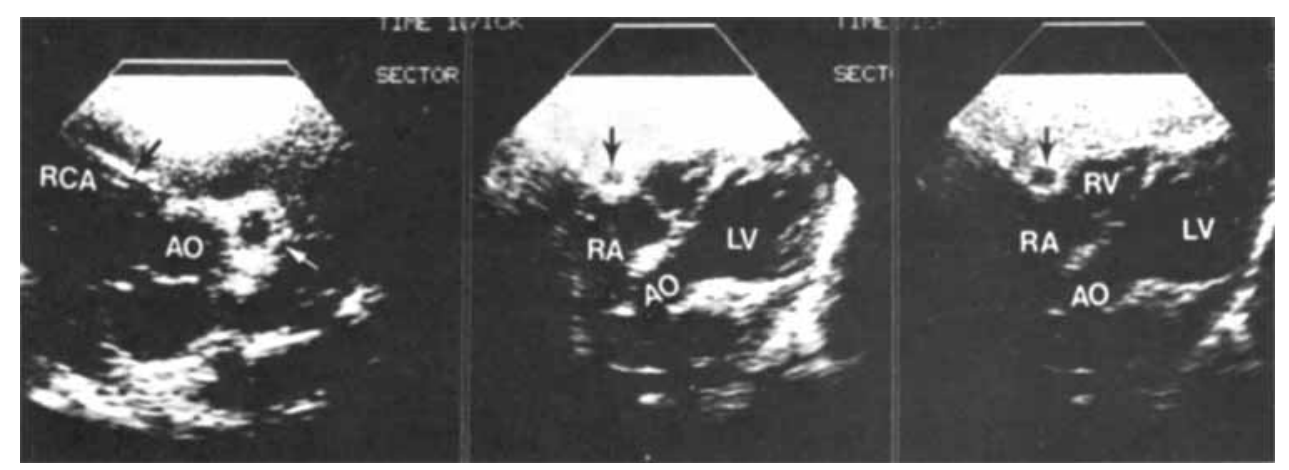

FIGURE 4. Echocardiogram: a parasternal short axis view (left) showed normal proximal right coronary artery (black arrow). An aneurysm of the left coronary artery was also seen (white arrow). A subcostal modified 4-chamber view (middle and right, black arrows) showed a circular echo-free space representing aneurysm of the distal right coronary artery. AO = aorta, RCA = right coronary artery, other abbreviations as in Fig. 1. 


\section{REFERENCES}

1. Kato H, Ichinose E, Yoshioka F, et al: Fate of coronary aneurysms in Kawasaki disease: Serial coronary angiography and long term follow up study. Am J Cardiol 49:1758, 1982.

2. Suma K, Takeuchi Y, Shiroma K, et al: Early and late postoperative studies in coronary arterial lesions resulting from Kawasaki's disease in children. $J$ Thoracic Cardiovascular Surgery 84:224, 1982.

3. Janos G, Schwartz D, Kaplan S: Prospective evaluation of patients with Kawasaki disease. Pediatr Cardiol 2:178, 1982.

4. Onouchi Z, Shimazu S, Kiyosawa N, et al: Aneurysms of the coronary arteries in Kawasaki disease-An angiographic study of 30 cases. Circulation 66:6, 1982.

5. Yoshikawa J, Yanagihara K, Owaki T, et al: Cross- sectional echocardiographic diagnosis of coronary artery aneurysms in patients with the mucocutaneous lymph node syndrome. Circulation 59:133, 1979.

6. Hiraishi S, Yashiro K, Kusano S: Noninvasive visualization of coronary arterial aneurysm in infants and young children with mucocutaneous lymph node syndrome with two dimensional echocardiography. Am J Cardiol 43:1225, 1979.

7. Chung KJ, Brandt L, Fulton DR, et al: Cardiac and coronary arterial involvement in infants and children from New England with mucocutaneous lymph node syndrome (Kawasaki Disease). Am $J$ Cardiol 50:136, 1982.

8. Yoshida H, Maeda T, Funabashi T, et al: Subcostal two-dimensional echocardiographic imaging of peripheral right coronary artery in Kawasaki disease. Circulation 65:956, 1982. 